\title{
Lower Extremity Strength and Mechanics During Jumping in Women With Patellofemoral Pain
}

\author{
John D. Willson and Irene S. Davis
}

\begin{abstract}
Context: Lower extremity (LE) weakness might be associated with altered mechanics during weight bearing in subjects with patellofemoral pain syndrome (PFPS). Objective: To analyze LE strength, mechanics, and the association between these variables among women with and without PFPS during a simulated athletic task. Design: Case control. Setting: Motion-analysis laboratory. Subjects: 20 women with PFPS and 20 healthy women. Main Outcome Measures: Peak isometric lateral trunk-flexion, hipabduction, hip external-rotation, knee-flexion, and knee-extension strength, as well as hip- and knee-joint excursions and angular impulses during single-leg jumps. Results: PFPS subjects produced less hip-abduction, hip external-rotation, and trunk lateralflexion force than the control group. The PFPS group also demonstrated greater hipadduction excursion and hip-abduction impulses. The association between the strength measurements and LE mechanics was low. Conclusions: Women with PFPS demonstrate specific weaknesses and altered LE mechanics. Weakness is not, however, highly correlated with observed differences in mechanics. Keywords: anterior knee pain, kinematics, kinetics, hip, patella
\end{abstract}

Patellofemoral pain syndrome (PFPS) is the most common lower extremity (LE) injury among individuals who present to outpatient sports-medicine physicians. ${ }^{1}$ Recent prospective studies of PFPS suggest that as many as 1 in 10 young adults who engage in structured physical activity will complain of PFPS. ${ }^{2}$ It is also reported to be nearly twice as prevalent among individuals who report to a running-injury clinic as any other orthopedic complaint and is especially common among active women., ${ }^{1,3}$

Several factors are suspected to contribute to the etiology of PFPS. Anatomical factors such as patella alta and hypoplasia of the medial patellar facet undoubtedly contribute. ${ }^{4} \mathrm{An}$ increased quadriceps angle and altered LE mechanics during weight-bearing activities are also believed to increase the risk for this injury. It is interesting that large quadriceps angles and certain LE kinematics during weightbearing activities are characteristic of females., 5 This might partially explain the gender bias for this injury. For example, females are reported to perform activities such as running and jumping with greater hip internal rotation and adduction than

Willson is with the Physical Therapy Program, University of Wisconsin-La Crosse, La Crosse, WI 54601. Davis is with the Dept of Physical Therapy, University of Delaware, Newark, DE 19711. 
matched male controls. ${ }^{5,6}$ In addition, recent studies suggest that women with PFPS demonstrate increased knee external rotation and hip adduction across a range of activities including single-leg squats, running, and single-leg jumps. ${ }^{7}$ Cadaver studies of simulated weight bearing with knee external rotation or hip adduction indicate that these altered kinematics increase retropatellar stress, which might exacerbate PFPS symptoms. ${ }^{8-11}$

It has been speculated that there is an association between decreased proximal strength and increased altered joint excursion among subjects with PFPS. For example, recent studies demonstrate significantly decreased hip abduction, hip external rotation, and lateral trunk strength among women with PFPS compared with a healthy female control group. ${ }^{12-15}$ This weakness relative to healthy subjects is suspected to increase the likelihood of greater pelvic drop, hip adduction, and internal-rotation excursion during dynamic activities. ${ }^{14,16}$ The previous studies did not analyze knee-flexion or -extension strength, however, which raises the question of whether the proximal weakness observed in these studies is a reflection of general LE weakness or if the weakness is specific to the hip and trunk.

Another question that remains is the association these static strength measures have with joint excursions thought to contribute to the exacerbation or etiology of PFPS symptoms. To date, no study has reported the association between hip strength and joint excursion in subjects with and without PFPS. Of previous studies presenting hip-strength and other kinematic data in subjects with PFPS, the results have been conflicting. ${ }^{12,17}$ In both previous studies, hip-abduction and external-rotation strength differences were observed between subjects with and without PFPS. Bolgla et al, ${ }^{12}$ however, reported similar average hip internalrotation, hip-adduction, and knee-valgus angles during the stance phase for both groups during a stair-stepping task, casting doubt on the association between hip weakness and altered LE kinematics in subjects with PFPS. On the other hand, Dierks et a ${ }^{17}$ reported a low association between hip-abduction isometric strength measures and peak hip-adduction angle among PFPS subjects at the start of a prolonged run but a strong correlation between these measures at the conclusion of the run.

Analysis of the association between LE-strength tests and hip- and knee-joint kinetics would also be valuable for rehabilitation professionals to better understand the cause of altered LE kinematics. The association between proximal strength and hip- and knee-joint kinetics has not been previously reported, however, for individuals with PFPS. Currently, rehabilitation professionals associate decreased hip-abductor or hip external-rotation strength with the tendency to produce decreased hip-abductor and hip external-rotator torque during weightbearing activities, resulting in increased hip-adduction or hip internal-rotation excursion. Altered LE kinematics is affected, however, by both muscle capacity and neurological factors (muscle-activation level, timing and duration of activation). Thus, it is conceivable that static clinical strength-test results have little association with muscle-torque production during functional activities. Further study of this potential relationship is necessary.

The purpose of this study was to compare LE strength, kinematics, and kinetics among women with and without PFPS during a simulated athletic activity: single-leg jumping. In addition, we aimed to test the association between strength, joint excursions, and joint moments during this highly demanding task. We 
hypothesized that women with PFPS would produce relatively less hip-abduction, external-rotation, lateral trunk-flexion, and knee-extension strength than a healthy control group. Women with PFPS were expected to demonstrate greater contralateral pelvic-drop, hip-adduction, hip internal-rotation, and knee-flexion excursion and decreased hip-abduction, hip external-rotation, and knee-extension joint moments throughout the activity. Finally, we hypothesized that there would be an inverse association between these strength measures and the corresponding joint excursions and joint moments during single-leg jumps.

\section{Methods}

The procedures for this study were approved by the University of Delaware institutional review board. All subjects who participated provided informed consent. Based on a sample-size calculation $(\alpha=.05, \beta=.20)$ using variability data from pilot studies of healthy females performing single-leg jumps and a $5^{\circ}$ difference between groups, 20 healthy women and 20 women with PFPS were included in the study. Women were chosen because PFPS appears to occur disproportionately in women relative to men. ${ }^{1}$ All subjects were 18 to 35 years of age. Each was required to report an activity level greater than or equal to $5 / 10$ on the Tegner activity scale (regular participation in recreational sports that require running or jumping). All pregnant subjects and subjects reporting cardiovascular pathology as indicated by a physical activity readiness questionnaire were excluded from participation. Females in the healthy control group were required to have been free of LE symptoms at rest or during running or jumping for the preceding 2 years.

Subjects complaining of PFPS were evaluated by a licensed physical therapist for additional inclusion criteria. These criteria included a verbal pain score of at least a 3 (moderate) on a 0- to 10-point scale during at least 2 activities such as squatting, prolonged sitting, ascending or descending stairs, running, or jumping. Symptoms were required to occur behind or adjacent to the patella and not solely at the iliotibial band, patellar tendon, or knee-joint line. In addition, these subjects complained of symptoms on compression of the patella into the femoral condyles or with pressure applied to either the medial or lateral posterior surface of the patella. PFPS symptoms were required to have had an insidious onset and have been present for a minimum of 2 months. Finally, subjects with PFPS scored less than $85 / 100$ on the anterior knee-pain scale. ${ }^{18}$ Fifteen points on this scale has been determined to be the minimum clinically important difference from "normal."19 Any subject who had experienced a traumatic injury to the knee joint within the preceding 6 months or presented with symptoms of a meniscus lesion or ligamentrelated pathology was excluded from participation. In cases of bilateral symptoms, the most symptomatic leg was chosen for analysis. The limb of the subjects in the healthy control group was chosen randomly.

Peak isometric force was calculated for the following muscle actions: lateral trunk flexion, hip abduction, hip external rotation, knee extension, and knee flexion (Figure 1). All test positions were based on those identified in the literature and were gravity resisted. ${ }^{20-24}$ We chose the test position $\left(30^{\circ}\right.$ knee flexion) for knee-extension strength used by Walsworth et $\mathrm{al}^{23}$ because we felt that this position better tests the ability of subjects to generate force at a knee-flexion angle 

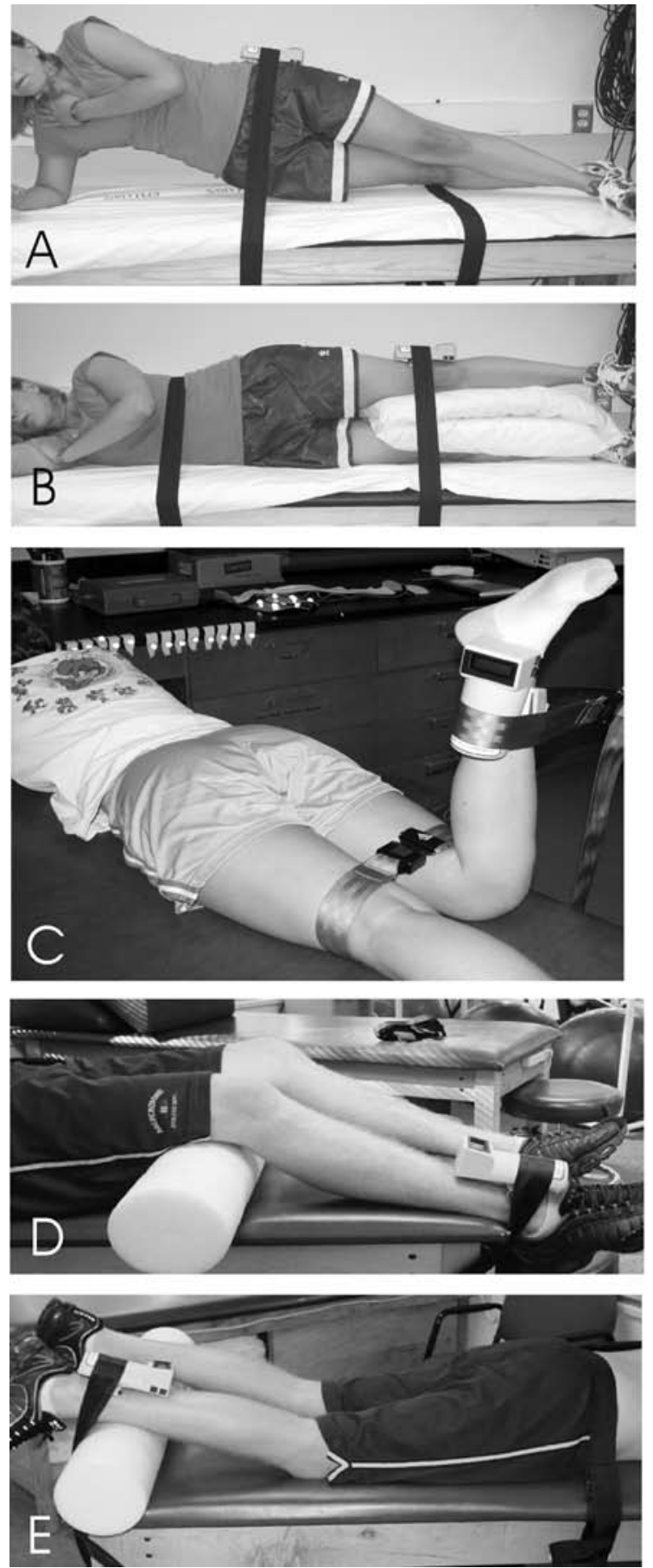

Figure 1 - Test positions used to measure (A) trunk lateral-flexion, (B) hip-abduction, (C) hip external-rotation, (D) knee-extension, and (E) knee-flexion strength. 
they would experience during single-leg jumps. Straps were used to stabilize the subject and handheld dynamometer (Lafayette Instruments, Lafayette, IN) to eliminate the influence of tester strength on these measurements. For each test, 1 practice and 3 experimental trials were performed for 5 seconds, with 15 seconds of rest between contractions. To facilitate comparison of our results with those of previous studies, strength measurements were normalized to subject body weight and multiplied by 100 . The average normalized peak-force values produced during the 3 trials was used for analysis. Before testing, within-rater reliability was determined for the principal investigator. Intraclass correlation coefficients (model 3,1) ranged from .93 to .97 for each test.

Three-dimensional LE mechanics were collected during single-leg jumps. Anatomical markers were placed over the greater trochanters, medial and lateral femoral condyles, medial and lateral tibial plateaus, medial and lateral malleoli, the first and fifth metatarsal heads, and the distal shoe. Additional tracking markers were positioned as clusters on the rear foot of the shoe, posterior lower leg, and lateral thigh. Pelvis-tracking markers were placed on the anterior superior iliac spine, iliac crest, and the L5-S1 interspace. Data were collected during a standing calibration trial, and the anatomical markers were removed. The tracking markers remained for all subsequent motion trials. All subjects performed testing wearing Nike Air Pegasus running shoes.

Subjects then performed 5 consecutive single-leg hops as high as possible on a force plate as their 3-dimensional marker coordinates were collected using a 6-camera Vicon 3D motion-analysis system (model MCAM 1, Vicon, Oxford Metrics, UK) at a sampling frequency of $120 \mathrm{~Hz}$. These coordinates were filtered using a fourth-order, zero-lag, low-pass Butterworth filter with a cutoff frequency of $10 \mathrm{~Hz}$. Kinetic ground-reaction-force data were collected at $1080 \mathrm{~Hz}$ (Bertec Corp, Worthington, $\mathrm{OH}$ ) and low-pass filtered at $50 \mathrm{~Hz}$ with a fourth-order, zerolag Butterworth filter. Jumping mechanics were analyzed during the 4 groundcontact phases beginning with the landing from the first jump until takeoff for the fifth jump. All subjects were given practice trials before testing until they reported they were comfortable with the task. Subjects were required to rest for 60 seconds before the data collection to minimize the potential influence of fatigue from the practice trials on LE mechanics. During this time, all subjects were asked to rate their knee pain after the 5 single-leg jumps using a 0 - to 10 -point verbal analog scale.

Visual3D (version 3.79, C-Motion Inc, Rockville, MD) was used to calculate kinematic and kinetic time-series data with each joint assigned 6 degrees of freedom. Customized computer programs (LabView 8.0.1, National Instruments, Austin, TX) were used to identify the discrete variables of interest from the timeseries data during the middle 4 jumps. These included transverse- and frontalplane hip-joint excursion, contralateral pelvic-drop excursion (with respect to the laboratory coordinate system), and knee-joint-flexion excursion.

This custom computer program also calculated hip transverse- and frontalplane and knee sagittal-plane kinetic data in the form of the joint angular impulse. The angular impulse is the sum of the net joint moment produced throughout an activity. This variable provides an estimate of the torque produced by muscle groups during the activity, as well as the torque from noncontractile tissue such as ligaments and articular surfaces. The angular impulse for this study was determined by integrating the hip- and knee-joint-moment curves over the stance phase 
for each individual single-leg jump. Joint-moment data were expressed as the internal joint moment relative to the distal segment.

Pelvis, hip-joint, and knee-joint excursions were defined as the change in joint or segment angle from the instance of ground contact for each jump to time of peak knee-extension moment. This is the time we expected the patellofemoral joint-reaction force to be greatest. Therefore, kinematic changes indentified in cadaver studies that reduce contact area between the femur and the patella might most significantly affect retropatellar stress at this time. Comparisons of strength, joint angular impulse, and joint and segment excursions were performed using independent $t$ tests with an alpha level set at .05 for all comparisons. Effect sizes were calculated to illustrate the magnitude of the difference between groups. Pearson correlation coefficients were determined for the association between the strength measures and joint excursions and joint angular impulses.

\section{Results}

Subjects from both groups were similar with respect to demographics and activity level (Table 1). No subject reported noteworthy pain during strength tests or single-leg jumps. Average (SD) pain ratings after single-leg jumps were $0.0(0.0)$ for the control group and $0.3(0.6)$ for subjects in the PFPS group. Significant differences between groups were found with respect to hip and trunk strength (Figure 2). Specifically, the PFPS group was $15 \%$ weaker in hip external rotation (effect size $[\mathrm{ES}]=.67, P=.04), 15 \%$ weaker in hip abduction $(\mathrm{ES}=.64, P=.05)$, and $29 \%$ weaker in lateral trunk flexion $(\mathrm{ES}=.76, P=.02)$. The difference between groups in knee-flexion (ES $=.03, P=.92)$ and knee-extension strength $(\mathrm{ES}=.36$, $P=.27$ ), however, was neither clinically nor statistically significant.

The PFPS group demonstrated greater hip-adduction excursion $(\mathrm{ES}=.65, P$ $=.05$ ) than the control group (Figures 3 and 4), but no other joint and segment excursions were statistically different among subjects with PFPS. Although many of the excursion variables were not statistically different between groups, moderate effect sizes were noted for greater contralateral pelvic-drop $(\mathrm{ES}=.45, P=.16)$ and knee-flexion excursion $(\mathrm{ES}=.49, P=.14$ ) among subjects with PFPS. A small effect size was noted for greater hip internal-rotation excursion $(\mathrm{ES}=.25, P$ $=.44$ ) for these subjects.

Kinetic differences between groups during single-leg jumps were also observed (Figures 4 and 5). The PFPS group produced an 18\% larger hip-abduction angular impulse than the control group ( $\mathrm{ES}=.62, P=.05)$. Although larger in the PFPS group, no difference was found between knee-extension $(\mathrm{ES}=.23, P=.49$ ) or

Table 1 Average Subject Demographics, Mean (SD)

\begin{tabular}{lcc}
\hline & Control & Patellofemoral pain \\
\hline Age $(\mathrm{y})$ & $23.7(3.6)$ & $23.3(3.1)$ \\
Height $(\mathrm{m})$ & $1.66(0.06)$ & $1.66(0.08)$ \\
Weight $(\mathrm{kg})$ & $61.1(5.4)$ & $61.7(10.6)$ \\
Tegner activity rating $(0-10)$ & $6.9(1.3)$ & $6.3(1.4)$ \\
Score on anterior knee-pain scale $(0-100)$ & $100.0(0.2)$ & $80.2(8.5)$ \\
\hline
\end{tabular}




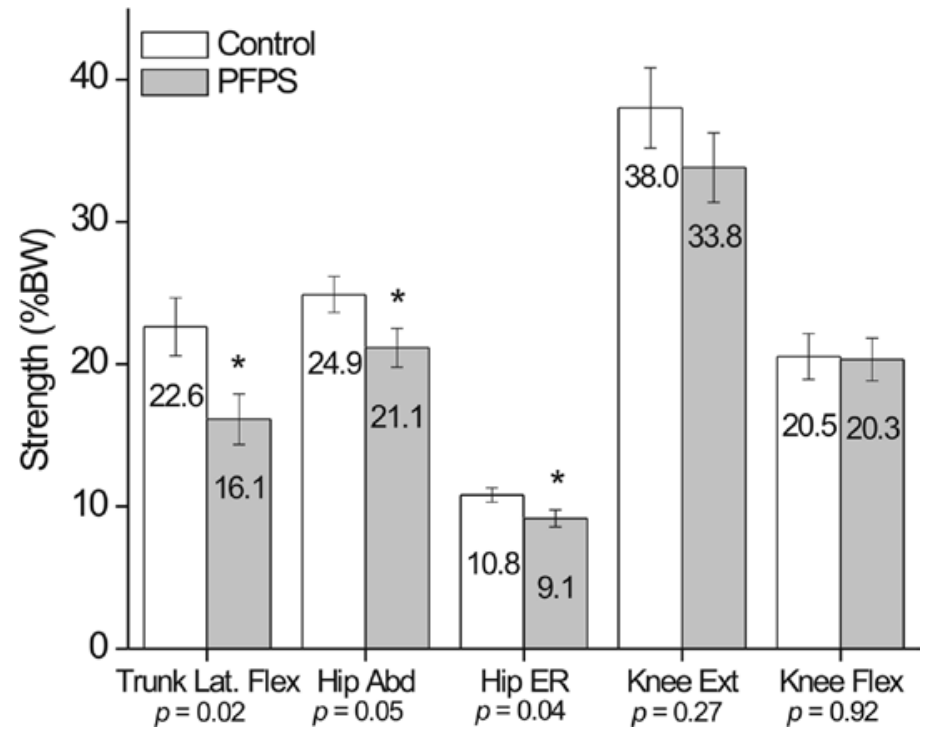

Figure 2 - Isometric strength for subjects with and without patellofemoral pain (PFPS). Error bars represent 1 SEM. Lat. = lateral; $\mathrm{Abd}=$ abduction; $\mathrm{ER}=$ external rotation; $\mathrm{Ext}=$ extension; Flex $=$ flexion. $* P<.05$.

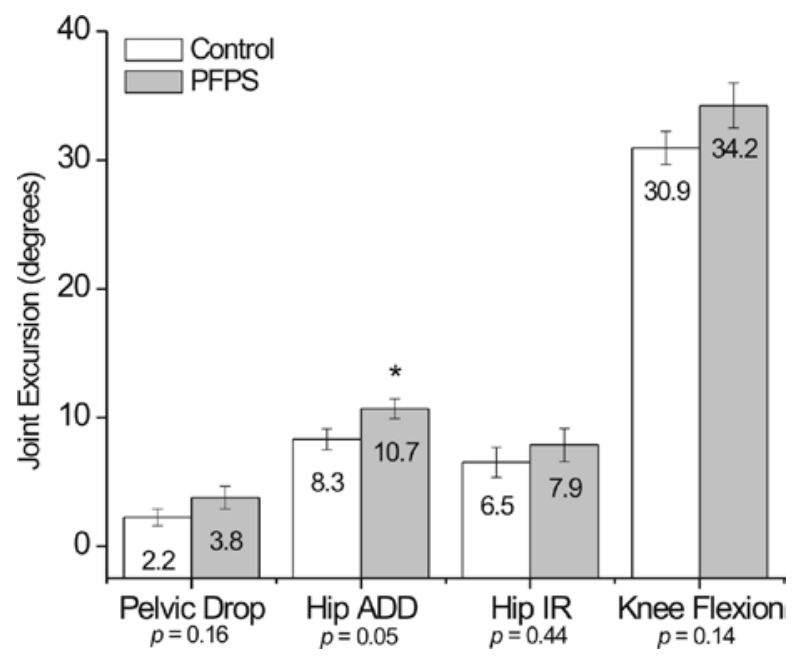

Figure 3 - Joint excursions for subjects with and without patellofemoral pain (PFPS) during single-leg jumping. Error bars represent 1 SEM. ADD = adduction; IR = internal rotation. $* P<.05$. 


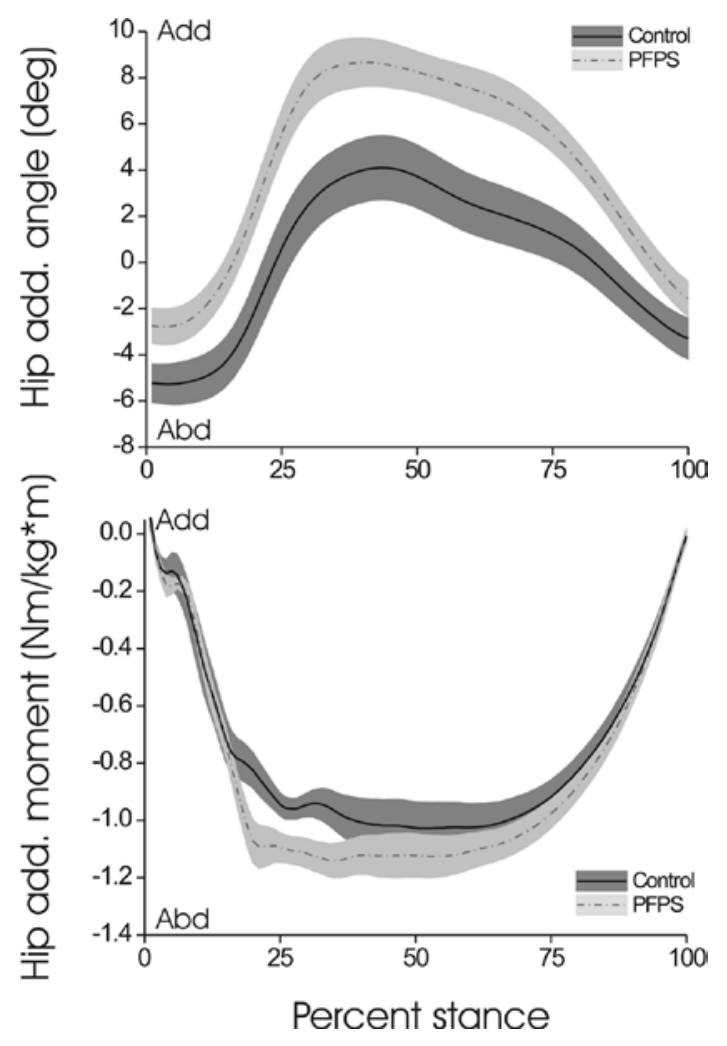

Figure 4 - Hip-adduction angle and joint-moment time-series composite curves for subjects with and without patellofemoral pain (PFPS) during single-leg jumping. The shaded area represents 1 SEM above and below the mean at each time point. Add = adduction; Abd $=$ abduction.

hip-rotation angular impulse (ES $=.26, P=.42$ ) between groups. Peak vertical ground-reaction forces were 2.9 times body weight in the control group compared with 2.7 times body weight for the PFPS group.

Correlations between strength measurements and LE mechanics were found to be low (Table 2). No statistically significant correlations between strength measurements and joint excursions during single-leg jumps were identified across all subjects or when the PFPS group was considered independently. The expected inverse association between decreased strength measurements and increased joint excursion was potentially clinically meaningful $(r=-.40, P=.10)$ for the hip frontal plane, but only when subjects in the PFPS were considered independently.

No statistically significant correlations were noted between strength measurements and joint angular impulses for all subjects (Table 3). The same was true among the PFPS group alone. Contrary to our expectations, all subjects demonstrated a positive (internal rotation) hip transverse-plane moment and hip 


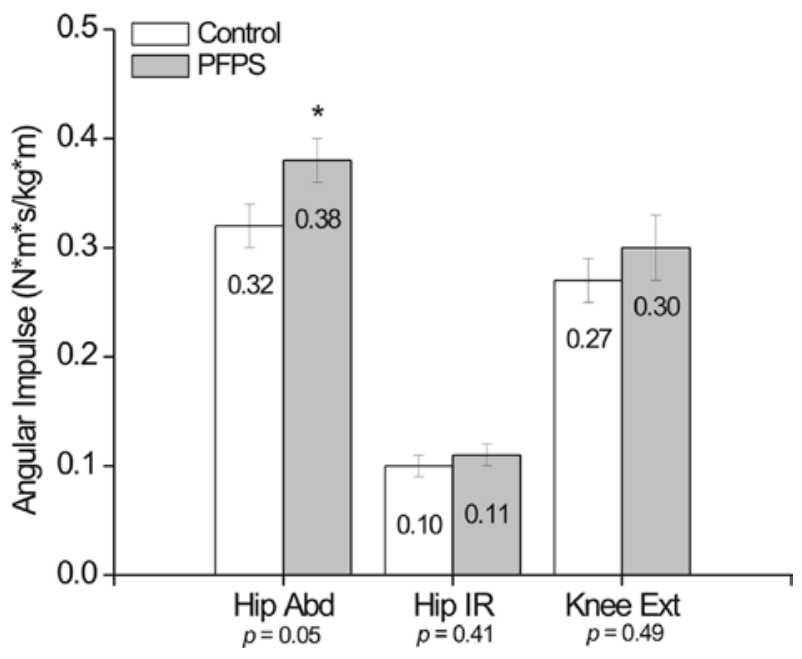

Figure 5 - Angular impulse for subjects with and without patellofemoral pain (PFPS) during single-leg jumping. Error bars represent $1 \mathrm{SEM}$. Abd = abduction; IR = internal rotation; Ext $=$ extension. $* P<.05$.

Table 2 Correlations ( $P$ Values) Between Strength and Joint and Segment Excursions, Mean (SD)

\begin{tabular}{lcc}
\hline Strength/excursion & All subjects & $\begin{array}{c}\text { Patellofemoral pain } \\
\text { only }\end{array}$ \\
\hline Side bridge/contralateral pelvic drop & $.05(.76)$ & $.07(.76)$ \\
Hip abduction/hip adduction & $-.04(.79)$ & $-.40(.10)$ \\
Hip external rotation/hip internal rotation & $-.12(.46)$ & $-.07(.78)$ \\
Knee extension/knee flexion & $.13(.44)$ & $.02(.93)$ \\
\hline
\end{tabular}

Table 3 Correlations ( $P$ Values) Between Strength and Joint Angular Impulse, Mean (SD)

\begin{tabular}{lcc}
\hline $\begin{array}{c}\text { Strength/Angular-impulse } \\
\text { correlation }\end{array}$ & All subjects & $\begin{array}{c}\text { Patellofemoral } \\
\text { pain only }\end{array}$ \\
\hline Hip abduction & $.08(.62)$ & $.06(.80)$ \\
Hip external rotation & $-.27(.09)$ & $-.36(.13)$ \\
Knee extension & $.31(.07)$ & $.25(.29)$ \\
\hline
\end{tabular}




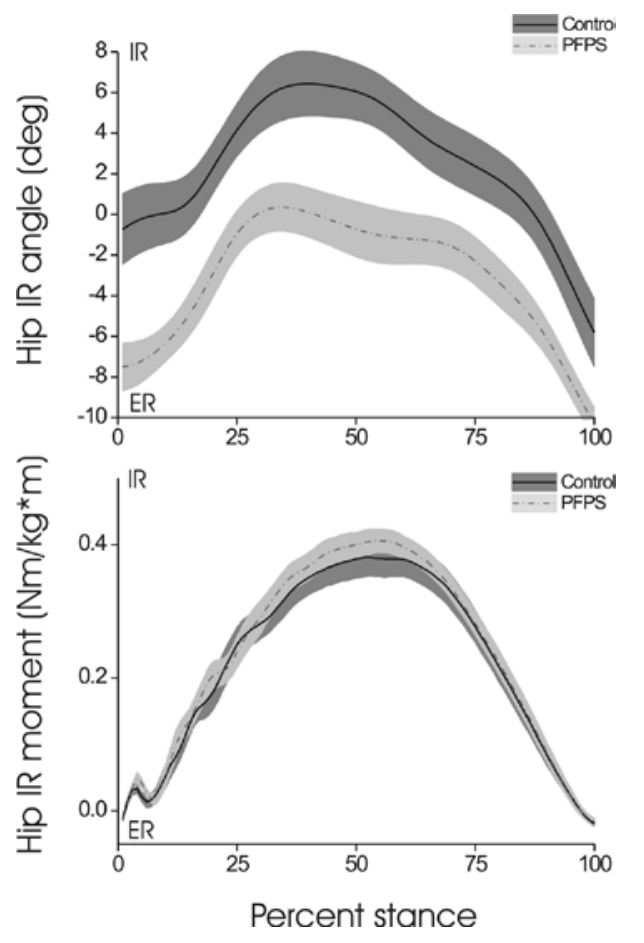

Figure 6 - Hip internal-rotation angle and joint-moment time-series composite curves for subjects with and without patellofemoral pain (PFPS) during single-leg jumping. The shaded area represents 1 SEM above and below the mean at each time point. IR = internal rotation; ER = external rotation.

internal-rotation joint excursion during the deceleration phase of single-leg landings. This might be interpreted as concentric action of the hip internal rotators during the first half of the activity followed by eccentric action of the internal rotators during the acceleration phase of the single-leg jump (Figure 6).

\section{Discussion}

The first aim of the current study was to analyze trunk-, hip-, and knee-joint strength between women with and without PFPS. Previous studies of hip-abduction and external-rotation strength in subjects with PFPS reported deficits ranging from $4 \%$ to $36 \%$ for hip external rotation and $14 \%$ to $27 \%$ for hip abduction in subjects with PFPS. ${ }^{12-15,17,22}$ The results of this study support the results of these previous studies, revealing strength deficits of $15 \%$ for both hip abduction and hip external rotation. In addition, subjects in the PFPS group produced 29\% less trunk lateral-flexion force than subjects in the control group. It is interesting to note that 
magnitude of the strength difference between groups for knee-flexion and -extension strength was neither clinically nor statistically significant. Therefore, it seems that women with PFPS do not have general LE weakness relative to their healthy counterparts. Rather, they demonstrate weakness in specific actions, perhaps underscoring the role of hip and trunk function in the etiology and exacerbation of PFPS.

Subjects with PFPS exhibited larger hip-adduction excursion than those in the control group. Hip adduction has previously been suggested to contribute to the etiology and exacerbation of PFPS.,14,16,25 Greater hip adduction during weight bearing might elongate the ipsilateral iliotibial band. This tension on the iliotibial band might lead to greater lateral force on the patella through the lateral patellar retinaculum. ${ }^{26,27}$ It is conceivable that this increase in lateral patellar force decreases retropatellar contact area and increases retropatellar stress. Repetitive exposure to such stress during weight-bearing activities might contribute to the etiology of these symptoms or exacerbation of preexisting complaints.

Hip internal-rotation excursion in the PFPS group was similar to that in the control group. The fact that transverse-plane hip kinematics were not different between groups despite differences in hip external-rotation strength is consistent with previous findings. ${ }^{12}$ Inspection of the time-series data for each group, however, reveals that the PFPS group performed single-leg jumping with less hip internal rotation throughout the activity (Figure 6). This result supports previous findings of decreased femoral internal rotation in subjects with PFPS during walking. ${ }^{28}$ Decreased femoral rotation was hypothesized to be a compensatory mechanism of subjects with PFPS, used to avoid large quadriceps angles and associated lateral retropatellar stress. We felt that, during more dynamic activities, subjects with PFPS would not be able to maintain such compensation strategies. Our results demonstrate that, even during this dynamic activity, the PFPS group continued to demonstrate decreased hip internal rotation.

It is interesting that the PFPS group produced larger hip-abduction moments through stance, resulting in larger angular impulses than in the healthy control group (Figure 4). Peak vertical ground-reaction force was similar between groups during the jump trials. Thus, the greater hip-abduction angular impulse is likely the result of a larger hip-abductor-muscle moment arm, perhaps as a result of the greater hip-adduction angle observed among subjects in the PFPS group. This result is in contrast to our hypothesis that individuals with decreased hipabduction strength would produce smaller hip-abduction moments, fostering increased hip-adduction excursion during single-leg jumps. Rather, if muscle torque is the product of muscle contractile capacity and neuromuscular activation parameters, this result suggests that hip-adduction excursion during single-leg jumps might be more closely related to hip-abductor neuromuscular factors such as activation level, onset time, or duration of the activation than to static hipabductor strength measurements. Future studies using electromyography are necessary to test this hypothesis.

A notable clinical application of this result among subjects with PFPS might be that rehabilitation strategies for increased hip-adduction excursion are more effective if they do not strictly focus on hip-abductor strengthening. Clearly, the cross-sectional nature of this study limits our ability to forecast the effectiveness of rehabilitation efforts to improve altered LE kinematics. Nonetheless, neuro- 
muscular reeducation techniques such as kinematic feedback during functional activities to promote even greater hip-abductor recruitment among subjects with PFPS might prove to be more effective in decreasing hip-adduction excursion thought to exacerbate PFPS symptoms than strengthening interventions alone. Recent studies of conservative management for PFPS have emphasized strengthening of hip and trunk musculature plus neuromuscular reeducation techniques with positive results. ${ }^{25}$

Unexpectedly, all subjects produced a hip internal-rotation angular impulse during single-leg jumps. Considered with respect to average hip-joint kinematics during the activity, this suggests that the hip internal rotators were acting concentrically during the landing of the single-leg jumps and concentrically during the jumping phase. Modeling studies suggest that a strong gluteus medius contraction with the hip flexed might produce a hip internal-rotation moment. ${ }^{29}$ Such strong gluteus medius contractions might not occur during bilateral jumping tasks because the contralateral leg stabilizes the pelvis in the frontal plane. Indeed, a previous report of hip transverse-plane moments during a bilateral landing and jumping task in healthy subjects indicated eccentric action of the hip internal rotators during the landing phase of the task. ${ }^{30}$ It is also possible that the intentional nature of repetitive single-leg jumping, constrained within the dimensions of the force plate, contributed to this result. Concentric hip internal rotation during the landing phase of the activity might be necessary to perform repetitive vertical jumping without lateral or rotational movement on the force plate.

Sagittal-plane knee-joint jumping kinematics and kinetics were not different between groups. Previous studies of subjects with PFPS during walking and stair climbing reported decreased knee-extension moment (KEM) in subjects with PFPS. ${ }^{31,32}$ Subjects in these studies were hypothesized to avoid elevated net KEM to minimize pain during the activity by decreasing gait velocity or perhaps by increasing forward trunk lean during the task. Other authors report decreased peak knee flexion as a mechanism to reduce KEM during walking. ${ }^{33}$ Our test simulated an athletic activity that was faster and associated with greater loading rates and peak external loads than those in the previous studies. Under these more dynamic conditions, the described compensation mechanisms to minimize KEMs might have been difficult to employ.

Decreased hip-abduction strength was moderately $(r=-.40)$ though not significantly correlated with increased hip-adduction excursion, but only for individuals with PFPS, during single-leg jumps. It is interesting to note that subjects with PFPS collectively had less hip-abduction strength than the control group. Previous authors have hypothesized that there is a threshold for strength below which the association between static strength measures and LE mechanical deviations during dynamic functional activities becomes apparent. ${ }^{17,34}$ Above this threshold, other factors such as variability in muscle-activation level might conceal the association between strength and kinematics. A greater proportion of subjects in the control group might have been above such a threshold. Thus, the significance of the association between these measures could have been reduced when all subjects were considered together.

Knee-extension strength was not strongly associated with knee-extension angular impulse or knee-flexion excursion $(.02<r<.31)$. Knee-extension strengthening is a common component of PFPS rehabilitation programs. These 
results suggest that increased knee-extension strength would not adversely affect knee-flexion excursion or knee-extension angular impulse, at least during singleleg jumping. Increased knee-flexion excursion or knee-extension angular impulse during weight bearing might increase retropatellar stress and exacerbate PFPS symptoms. Future study is necessary to further explore the impact of kneeextension strengthening on LE mechanics that influence the patellofemoral joint.

There are strengths and limitations to this study. Joint-excursion data have not been previously reported for subjects with and without PFPS during a dynamic activity such as single-leg jumping. Joint excursion is less sensitive to discrepancies in marker placement for kinematic analysis or structural differences among groups that can affect average and peak joint-angle values in previous studies yet have no intuitive relationship with peak isometric strength. In addition, this study presents hip- and knee-joint kinetic data, which further clarify the demands placed on the muscles of interest during this activity. Limitations of this study include the fact that isometric strength tests might not provide the best measure of a muscle's functional capacity. Indeed, it is well known that muscles produce greater force during eccentric contractions. Furthermore, the vast majority of contractions during the deceleration phase of a jump are eccentric in nature. Therefore, it is possible that the association between these LE mechanics and LE muscle-strength measures might be greater using isokinetic eccentric strength tests. In addition, other measures such as timing and level of muscle recruitment, as well as cocontraction patterns, might provide more insight into these relationships. Finally, subjects were not screened for a history of low back injury, which might be a confounding factor in the interpretation of lateral trunk-flexion-strength group differences.

\section{Acknowledgments}

Support for this project was provided by the Foundation for Physical Therapy.

\section{References}

1. Taunton JE, Ryan MB, Clement DB, McKenzie DC, Lloyd-Smith DR, Zumbo BD. A retrospective case-control analysis of 2002 running injuries. Br J Sports Med. 2002;36:95-101.

2. Witvrouw E, Lysens R, Bellemans J, Cambier D, Vanderstraeten G. Intrinsic risk factors for the development of anterior knee pain in an athletic population. A two-year prospective study. Am J Sports Med. 2000;28:480-489.

3. DeHaven KE, Lintner DM. Athletic injuries: comparison by age, sport, and gender. Am J Sports Med. 1986;14:218-224.

4. Dixit S, DiFiori JP, Burton M, Mines B. Management of patellofemoral pain syndrome. Am Fam Physician. 2007;75:194-202.

5. Ferber R, Davis IM, Williams DS III. Gender differences in lower extremity mechanics during running. Clin Biomech (Bristol, Avon). 2003;18:350-357.

6. Lephart SM, Ferris CM, Riemann BL, Myers JB, Fu FH. Gender differences in strength and lower extremity kinematics during landing. Clin Orthop Relat Res. 2002;401:162-169.

7. Willson JD, Davis IS. Lower extremity mechanics of females with and without patellofemoral pain across activities with progressively greater task demands. Clin Biomech (Bristol, Avon). 2008;23:203-211. 
8. Csintalan RP, Schulz MM, Woo J, McMahon PJ, Lee TQ. Gender differences in patellofemoral joint biomechanics. Clin Orthop Relat Res. 2002;402:260-269.

9. Lee TQ, Morris G, Csintalan RP. The influence of tibial and femoral rotation on patellofemoral contact area and pressure. J Orthop Sports Phys Ther. 2003;33:686-693.

10. Li G, DeFrate LE, Zayontz S, Park SE, Gill TJ. The effect of tibiofemoral joint kinematics on patellofemoral contact pressures under simulated muscle loads. J Orthop Res. 2004;22:801-806.

11. Ostermeier S, Holst M, Bohnsack M, Hurschler C, Stukenborg-Colsman C, Wirth CJ. Dynamic measurement of patellofemoral contact pressure following reconstruction of the medial patellofemoral ligament: an in vitro study. Clin Biomech (Bristol, Avon). 2007;22:327-335.

12. Bolgla LA, Malone TR, Umberger BR, Uhl TL. Hip strength and hip and knee kinematics during stair descent in females with and without patellofemoral pain syndrome. J Orthop Sports Phys Ther. 2008;38:12-18.

13. Robinson RL, Nee RJ. Analysis of hip strength in females seeking physical therapy treatment for unilateral patellofemoral pain syndrome. J Orthop Sports Phys Ther. 2007;37:232-238.

14. Ireland ML, Willson JD, Ballantyne BT, Davis IM. Hip strength in females with and without patellofemoral pain. J Orthop Sports Phys Ther. 2003;33:671-676.

15. Willson JD, Binder-Macleod S, Davis IS. Lower extremity jumping mechanics of female athletes with and without patellofemoral pain before and after exertion. Am J Sports Med. 2008;36:1587-1596.

16. Powers CM. The influence of altered lower-extremity kinematics on patellofemoral joint dysfunction: a theoretical perspective. J Orthop Sports Phys Ther. 2003;33:639646.

17. Dierks TA, Manal KT, Hamill J, Davis IS. Proximal and distal influences on hip and knee kinematics in runners with patellofemoral pain during a prolonged run. J Orthop Sports Phys Ther. 2008;38:448-456.

18. Kujala UM, Jaakkola LH, Koskinen SK, Taimela S, Hurme M, Nelimarkka O. Scoring of patellofemoral disorders. Arthroscopy. 1993;9:159-163.

19. Watson CJ, Propps M, Ratner J, Zeigler DL, Horton P, Smith SS. Reliability and responsiveness of the lower extremity functional scale and the anterior knee pain scale in patients with anterior knee pain. J Orthop Sports Phys Ther. 2005;35:136-146.

20. Bohannon RW. Reference values for extremity muscle strength obtained by hand-held dynamometry from adults aged 20 to 79. Arch Phys Med Rehabil. 1997;78:26-31.

21. McGill SM, Childs A, Liebenson C. Endurance times for low back stabilization exercises: clinical targets for testing and training from a normal database. Arch Phys Med Rehabil. 1999;80:941-944.

22. Piva SR, Goodnite EA, Childs JD. Strength around the hip and flexibility of soft tissues in individuals with and without patellofemoral pain syndrome. J Orthop Sports Phys Ther. 2005;35:793-801.

23. Walsworth M, Schneider R, Schultz J, et al. Prediction of 10 repetition maximum for short-arc quadriceps exercise from hand-held dynamometer and anthropometric measurements. J Orthop Sports Phys Ther. 1998;28:97-104.

24. Worrell TW, Karst G, Adamczyk D, et al. Influence of joint position on electromyographic and torque generation during maximal voluntary isometric contractions of the hamstrings and gluteus maximus muscles. $J$ Orthop Sports Phys Ther. 2001;31:730-740.

25. Mascal CL, Landel R, Powers C. Management of patellofemoral pain targeting hip, pelvis, and trunk muscle function: 2 case reports. J Orthop Sports Phys Ther. 2003;33:647-660.

26. Puniello MS. Iliotibial band tightness and medial patellar glide in patients with patellofemoral dysfunction. J Orthop Sports Phys Ther. 1994;17:144-148. 
27. Wu CC, Shih $\mathrm{CH}$. The influence of iliotibial tract on patellar tracking. Orthopedics. 2004;27:199-203.

28. Powers CM, Chen PY, Reischl SF, Perry J. Comparison of foot pronation and lower extremity rotation in persons with and without patellofemoral pain. Foot Ankle Int. 2002;23:634-640.

29. Delp SL, Hess WE, Hungerford DS, Jones LC. Variation of rotation moment arms with hip flexion. J Biomech. 1999;32:493-501.

30. Chaudhari AM, Lindenfeld TN, Andriacchi TP, et al. Knee and hip loading patterns at different phases in the menstrual cycle: implications for the gender difference in anterior cruciate ligament injury rates. Am J Sports Med. 2007;35:793-800.

31. Heino Brechter J, Powers CM. Patellofemoral stress during walking in persons with and without patellofemoral pain. Med Sci Sports Exerc. 2002;34:1582-1593.

32. Salsich GB, Brechter JH, Powers CM. Lower extremity kinetics during stair ambulation in patients with and without patellofemoral pain. Clin Biomech (Bristol, Avon). 2001;16:906-912.

33. Nadeau S, Gravel D, Hebert L, Arsenault AB, Lepage Y. Gait study of patients with patellofemoral pain syndrome. Gait Posture. 1997;5:21-27.

34. Fosang A, Baker R. A method for comparing manual muscle strength measurements with joint moments during walking. Gait Posture. 2006;24:406-411.

STATEMENT OF OWNERSHIP, MANAGEMENT, AND CIRCULATION OF JOURNAL OF SPORT REHABILITATION (ISSN 1056-6716), as required by 39 U.S. Code 3685 :

The Journal of Sport Rehabilitation (ISSN 1056-6716) is published four times a year (quarterly). Subscription fees are $\$ 60$ per year for individuals and $\$ 240$ per year for institutions.

The owner of the Journal of Sport Rehabilitation is Human Kinetics, Inc., whose office of publication is at 1607 N. Market St., Champaign, IL 61820-2200. The editor is Carl G. Mattacola, Division of Athletic Training, University of Kentucky, Lexington, KY 40536. There are no bondholders, mortgagees, or other security holders.

Average number of copies printed per issue (net press run) during the preceding 12 months is 877; number of copies nearest to filing date is 840 . Average number of copies of each issue distributed after mass mailing to subscribers during the preceding 12 months is 11 ; number of copies nearest to filing date is 12 . Average number of copies of each issue distributed in mass mailing to subscribers during the preceding 12 months is 566; number of copies nearest to filing date is 566 . Average number of copies of each issue distributed free during the preceding 12 months is 43 ; number of copies nearest to filing date is 46 . 
Copyright of Journal of Sport Rehabilitation is the property of Human Kinetics Publishers, Inc. and its content may not be copied or emailed to multiple sites or posted to a listserv without the copyright holder's express written permission. However, users may print, download, or email articles for individual use. 